\title{
Chemical Reaction and Thermal Diffusion Effects on Mass Transfer Flow through an Inclined Plate
}

\author{
Farjana Akter1, Md. Manjiul Islam¹, Ariful Islam1, Md. Shakhaoath Khan², \\ Md. Saddam Hossain ${ }^{1}$ \\ ${ }^{1}$ Mathematics Discipline, Khulna University, Khulna, Bangladesh \\ ${ }^{2}$ Discipline of Chemical Engineering, University of Newcastle, Callaghan, Australia \\ Email: munni091211@gmail.com, manjiul.math@gmail.com, arif@math.ku.ac.bd \\ mdshakhaoath.khan@uon.edu.au, ronykumath@gmail.com
}

Received 6 February 2016; accepted 26 March 2016; published 29 March 2016

Copyright (C) 2016 by authors and Scientific Research Publishing Inc.

This work is licensed under the Creative Commons Attribution International License (CC BY).

http://creativecommons.org/licenses/by/4.0/

c) (i) Open Access

\begin{abstract}
A numerical investigation of boundary layer mass transfer flow through an inclined plate with the effect of chemical reaction and thermal diffusion is presented in this study. The governing partial differential equations (PDE) are transformed to a system of dimensionless non-similar coupled PDEs. The transformed, non-similar conservations equations (momentum balance equation, energy balance equation and concentration balance equation) are then solved using a numerical approach known as explicit finite difference method (EFDM). Basically EFDM introduced for the unsteadiness in the momentum, temperature, and concentration fluid fields is based on the time dependent fluid velocity, temperature and concentration of the boundary surface. During the course of discussion, it is found that the various parameters related to the problem influence the calculated resultant expressions. The computed numerical solution results for the velocity, temperature, and concentration distribution with the effect of various important dimensionless parameters (Grashof number, Modified Grashof number, Prandtl number, Schmidt number, Soret number, Dufour number, chemical reaction parameter and inclination parameter) entering into the problems are critically analyzed and discussed graphically. It can be seen that two physical phenomena chemical reaction and thermal diffusion can greatly effect on the boundary layer fluid flows through an inclined plate.
\end{abstract}

\section{Keywords}

Chemical Reaction, Mass Transfer, Inclined Plate, Soret Effects, Dufour Effects

How to cite this paper: Akter, F., Islam, Md.M., Islam, A., Khan, Md.S. and Hossain, Md.S. (2016) Chemical Reaction and Thermal Diffusion Effects on Mass Transfer Flow through an Inclined Plate. Open Journal of Fluid Dynamics, 6, 62-74. 


\section{Introduction}

The effect of thermal diffusion and chemical reaction on heat and mass transfer fluid flow has a great importance to the engineering community and to investigators dealing with the problems in many industrial processes and technological fields which experience not only temperature difference but also concentration difference. The concentration difference may sometimes produce quantitative and qualitative changes to the rate of heat transfer. Some industrial applications are found in food drying, food processing or wood and paper production. Besides, the characteristics of the flow of blood through arteries and veins are of considerable medical interest. In view of the above interests, a series of investigations have been made by different scholars. Pera and Gebhart [1] were the first authors to investigate and combine buoyancy effects of thermal and mass diffusion on natural conviction flow. Soundalgekar and Ganesan [2] presented a finite difference analysis of unsteady natural convective flow past a semi-infinite plate with mass transfer. Hydro-magnetic heat and mass transfer in flow of a viscous incompressible fluid past an infinite vertical porous plate was analyzed by Singh et al. [3]. Using a finite difference technique, an unsteady free convective heat and mass transfer flow from an infinite vertical plate with constant suction have been numerically investigated by Ambethkar [4]. Recently Sivaiah et al. [5] studied heat and mass transfer effects on MHD free convective flow past a vertical porous plate.

The problem of mass transfer flow through an inclined plate has generated much interest from astrophysical, renewable energy system and also hypersonic aerodynamics researchers for a number of decades. Umemura and Law [6] developed a generalized formulation for the natural convection boundary layer flow over a flat plate with arbitrary inclination. They found that the flow characteristics depend not only on the extent of inclination but also on the distance from the leading edge. Chamkha and Khaled [7] investigated the problem of coupled heat and mass transfer by hydromagnetic free convection from an inclined plate in the presence of internal heat generation or absorption, and similarity solutions were presented. Reddy and Reddy [8] performed an analysis to study the natural convection flow over a permeable inclined surface with variable temperature, momentum and concentration. Singh [9] studied heat and mass transfer in MHD boundary layer flow past an inclined plate with viscous dissipation in porous medium. Micropolar fluid behavior on MHD free convection and mass transfer with constant heat and mass fluxes is studied numerically by Ali et al. [10]. Recently Islam et al. [11] have studied mass transfer flow through an inclined plate with porous medium.

In many transport processes existing in nature and in industrial applications in which heat and mass transfer is a consequence of buoyancy effects caused by diffusing of heat and chemical species. The study of such process is useful for improving a number of chemical technologies, such as polymer production, enhanced oil recovery, underground energy transport, manufacturing of ceramic and food processing. In many hydrometallurgical and chemical technology industries, heat and mass transfer is of considerable importance due to chemical reaction effects. Basically chemical reaction takes place between an external mass and the fluid in many chemical engineering processes. It can be designated as either a mixed or stable process, which be contingent on whether it occurs at a boundary or as a single-phase volume reaction. Chaudhary and Jha [12] studied the effect of chemical reaction on MHD micropolar fluid flow past a vertical plate in slip flow regime. Effects of mass transfer with a chemical reaction on unsteady flow past an accelerated isothermal vertical plate are investigated by Muthuchumaraswamy et al. [13]. Rajesh et al. [14] have discussed the effects of chemical reaction and radiation effects on MHD flow past an infinite vertical plate with variable temperature. The importance of thermal-diffusion and diffusion-thermo effects for various fluid flows has been studied by Eckert and Drake [15]. Olajuwon [16] examined convection heat and mass transfer in a hydromagnetic flow of a second grade fluid past a semi-infinite streching sheet in the presence of thermal diffusion and thermal radiation. Kumar et al. [17] have investigated thermal diffusion and radiation effects on unsteady MHD flow through porous medium with variable temperature and mass diffusion in the presence of heat source or sink.

There have been few studies on heat transfer boundary layer flow in the literature however mass transfer flow through an inclined plate with the effect of chemical reaction and thermal diffusion effects still need more attraction to the researchers [18] [19].

The objective of this research is to investigate the fluid behavior with the effect of chemical reaction and thermal diffusion on mass transfer flow in an inclined plate. In this research the governing equations of the problem contain a system of partial differential equations which are transformed by usual transformation into a non-dimensional system of partial coupled non-linear differential equations. The obtained non-similar partial differential equations are solved numerically by EFDM. The results of this research are discussed for the differ- 
ent values of the well-known dimensionless parameters and are shown graphically.

\section{Formulation and Solution}

\subsection{Mathematical Model of Flow}

By introducing Cartesian co-ordinate system, the $\mathrm{X}$-axis is chosen along the plate in the direction of the flow and the Y-axis is normal to it. The physical configuration has been shown in Figure 1. Initially it has been considered that the plate as well as the fluid is at the same temperature $T\left(T_{\infty}\right)$ and concentration level $C\left(C_{\infty}\right)$ everywhere in the fluid is same. Also it is considered that the fluid and the plate is at rest after that the plate is to be moving with a constant velocity $U_{0}$ in its own plane and instantaneously at time $t>0$ the species concentration and the temperature of the plate are raised to $C_{w}\left(>C_{\infty}\right)$ and $T_{w}\left(>T_{\infty}\right)$, which are there after maintained constant, where $C_{w}, T_{w}$ are species concentration and temperature at the wall of the plate and $C_{\infty}, T_{\infty}$ are the concentration of the species far away from the plate. Within the framework of the above stated assumptions with reference to the generalized equations described before the equation relevant to the transient two dimensional problems are governed by the following system of coupled non-linear differential equations using the Boussinesq and boundary layer approximations.

\section{Continuity Equation}

$$
\frac{\partial u}{\partial x}+\frac{\partial v}{\partial y}=0
$$

\section{Momentum Equation}

$$
\frac{\partial u}{\partial t}+u \frac{\partial u}{\partial x}+v \frac{\partial u}{\partial y}=g \beta_{T}\left(T-T_{\infty}\right) \cos \alpha+g \beta_{C}\left(C-C_{\infty}\right) \cos \alpha+v\left(\frac{\partial^{2} u}{\partial y^{2}}\right) .
$$

\section{Energy Equation}

$$
\frac{\partial T}{\partial t}+u \frac{\partial T}{\partial x}+v \frac{\partial T}{\partial y}=\frac{K}{\rho C_{p}} \frac{\partial^{2} T}{\partial y^{2}}+D_{m} \frac{\partial^{2} c}{\partial y^{2}} .
$$

\section{Concentration Equation}

$$
\frac{\partial C}{\partial t}+u \frac{\partial C}{\partial x}+v \frac{\partial C}{\partial y}=D_{m} \frac{\partial^{2} C}{\partial y^{2}}+D_{T} \frac{\partial^{2} T}{\partial y^{2}}-K_{C}\left(C-C_{\infty}\right) .
$$

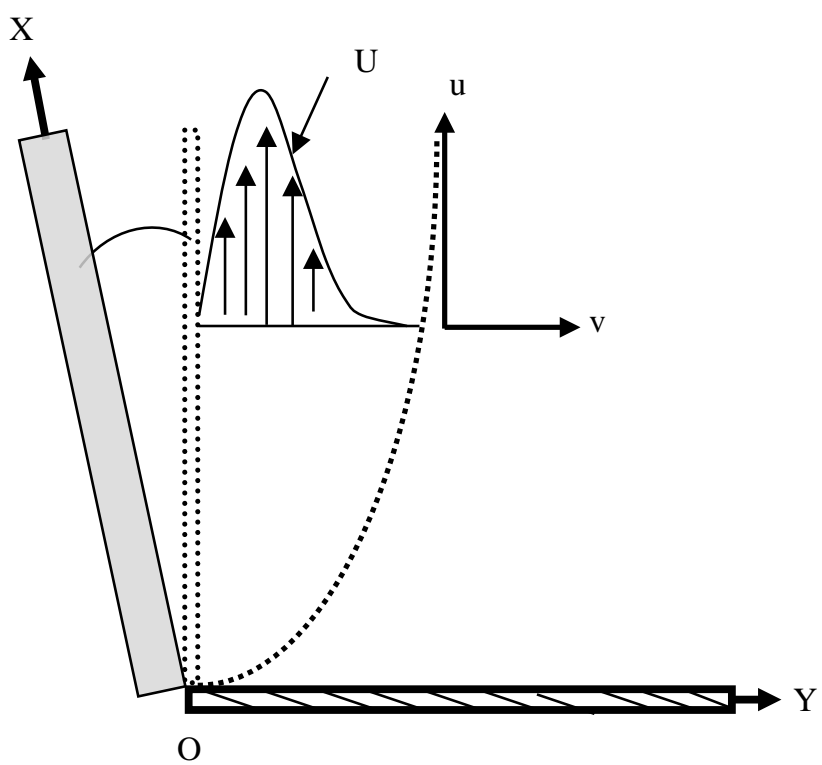

Figure 1. The Physical model and coordinate system. 
With the corresponding initial and boundary conditions are

At

$$
\begin{array}{ll}
t=0 \quad u=0, v=0, T \rightarrow T_{\infty}, C \rightarrow C_{\infty} \text { everywhere } . \\
u=0, v=0, T \rightarrow T_{\infty}, C \rightarrow C_{\infty} \text { at } x=0 \\
t>0 \quad u=U_{0}, v=0, T \rightarrow T_{w}, C \rightarrow C_{w} \text { at } y=0 . \\
u=0, v=0, T \rightarrow T_{\infty}, C \rightarrow C_{\infty} \text { at } y \rightarrow \infty
\end{array}
$$

where $x, y$ are Cartesian co-ordinate, $u, v$ are the components of flow velocity along $x, y$ directions respectively. Here $g$ is the local acceleration due to gravity; $v$ is the kinetic viscosity; $\rho$ is the density of the fluid, $D_{m}$ is the coefficient of mass diffusivity, $D_{T}$ is the coefficient of Thermal diffusivity and $K_{C}$ be the chemical reaction parameter.

\subsection{Mathematical Formulation}

Since the solution of the governing equations under the initial and boundary conditions will be based on a finite difference method it is required to make the said equations dimensionless.

For this purpose it has been now introduced the following dimensionless variables;

$$
\begin{aligned}
& X=\frac{x U_{0}}{v}, Y=\frac{y U_{0}}{v}, U=\frac{u}{U_{0}}, V=\frac{v}{U_{0}}, \\
& \tau=\frac{t U_{0}^{2}}{v}, \bar{T}=\frac{T-T_{\infty}}{T_{w}-T_{\infty}} \text { and } \bar{C}=\frac{C-C_{\infty}}{C_{w}-C_{\infty}}
\end{aligned}
$$

where $\tau$ represents the dimensionless time, $X \& Y$ be the dimensionless Cartesian coordinates, where $U$ and $V$ be the dimensionless velocity components, $T$ and $C$ is the dimensionless temperature and concentration respectively.

Using the above relation, we obtain the following non-dimensional coupled partial differential equation,

$$
\begin{aligned}
& \frac{\partial U}{\partial X}+\frac{\partial V}{\partial Y}=0 \\
& \frac{\partial U}{\partial \tau}+U \frac{\partial U}{\partial X}+V \frac{\partial U}{\partial Y}=\frac{\partial^{2} U}{\partial Y^{2}}+G_{r} \bar{T} \cos \alpha+G_{m} \bar{C} \cos \alpha \\
& \frac{\partial \bar{T}}{\partial \tau}+U \frac{\partial \bar{T}}{\partial X}+V \frac{\partial \bar{T}}{\partial Y}=\frac{1}{P_{r}} \frac{\partial^{2} \bar{T}}{\partial Y^{2}}+D_{f} \frac{\partial^{2} \bar{C}}{\partial Y^{2}} \\
& \frac{\partial \bar{C}}{\partial \tau}+U \frac{\partial \bar{C}}{\partial X}+V \frac{\partial \bar{C}}{\partial Y}=\frac{1}{S_{C}} \frac{\partial^{2} \bar{C}}{\partial Y^{2}}+S_{o} \frac{\partial^{2} \bar{T}}{\partial Y^{2}}-K_{r} \bar{C}
\end{aligned}
$$

where,

$$
\begin{aligned}
& G_{r}=v g \beta_{T} \frac{\left(T_{w}-T_{\infty}\right)}{U_{0}^{3}} \text { (Grashof Number), } \\
& G_{m}=v g \beta_{C} \frac{\left(C_{w}-C_{\infty}\right)}{U_{0}^{3}} \text { (Modified Grashof Number), } \\
& P_{r}=\frac{v \rho C_{p}}{K} \text { (Prandtl Number), } \\
& S_{C}=\frac{v}{D_{m}} \text { (Schmidt Number), } \\
& S_{o}=\frac{D_{T}\left(T_{w}-T_{\infty}\right)}{v\left(C_{w}-C_{\infty}\right)} \text { (Soret Number), }
\end{aligned}
$$


$D_{f}=\frac{D_{m}\left(C_{w}-C_{\infty}\right)}{v\left(T_{w}-T_{\infty}\right)}$ (Dufour Number),

$K_{r}=\frac{K_{C} U}{U_{0}^{2}}$ (Chemical Reaction Parameter).

Also the associate initial and boundary condition become

At

$$
\begin{array}{cl}
\tau=0 & U=0, V=0, \bar{T}=0, \bar{C}=0 \text { everywhere. } \\
& U=0, V=0, \bar{T}=0, \bar{C}=0 \text { at } X=0 \\
\tau>0 & U=1, V=0, \bar{T}=1, \bar{C}=1 \text { at } Y=0 \\
& U=0, V=0, \bar{T}=0, \bar{C}=0 \text { at } Y \rightarrow \infty
\end{array}
$$

\subsection{Numerical Solution}

The explicit finite difference method (EFDM) has been used to solve the governed second order nonlinear coupled dimensionless partial differential equations with the corresponding initial and boundary conditions. To obtain a system of finite difference equations, the flow region is divided into a grid or meshes of lines parallel to $X$ and $Y$ axes where $X$-axis is taken along the plate and $Y$-axis is normal to the plate. Here it is considered that $X_{\max }(=400)$ i.e. $X$ varies from 0 to 400 and regarding $Y_{\max } \quad(=25)$ as corresponding to $Y \rightarrow \infty$ i.e. $Y$ varies from 0 to 25. It is also considered that $m=60$ and $n=60$ grid spacing in $X$ and $Y$ directions respectively. We have the constant mesh size along $X$ direction, $\Delta X=6.67(0 \leq X \leq 400)$ and the constant mesh size along $Y$ direction, $\Delta Y=0.42(0 \leq Y \leq 25)$ with the smaller time-step $\Delta \tau=0.05$

Let $U^{\prime}, \bar{C}^{\prime}$ and $\bar{T}^{\prime}$ denote the values of $U, \bar{C}$ and $\bar{T}$ at the end of a time-step respectively. Using the finite difference approximations we obtain the following set of finite difference equations,

$$
\begin{aligned}
& \frac{U_{i, j}-U_{i-1, j}}{\Delta X}+\frac{V_{i, j}-V_{i, j-1}}{\Delta Y}=0 . \\
& \frac{U_{i, j}^{\prime}-U_{i, j}}{\Delta \tau}+U_{i, j} \frac{U_{i, j}-U_{i-1, j}}{\Delta X}+V_{i, j} \frac{U_{i, j+1}-U_{i, j}}{\Delta Y}=G_{r} \bar{T}_{i, j} \cos \alpha+G_{m} \bar{C}_{i, j} \cos \alpha+\frac{U_{i, j+1}-2 U_{i, j}+U_{i, j-1}}{(\Delta Y)^{2}} . \\
& \frac{\bar{T}_{i, j}^{\prime}-T_{i, j}}{\Delta \tau}+U_{i, j} \frac{\bar{T}_{i, j}-\bar{T}_{i-1, j}}{\Delta X}+V_{i, j} \frac{\bar{T}_{i, j+1}-\bar{T}_{i, j}}{\Delta Y}=\frac{1}{P_{r}} \frac{\bar{T}_{i, j+1}-2 \bar{T}_{i, j}+\bar{T}_{i, j-1}}{(\Delta Y)^{2}}+D_{f} \frac{\bar{C}_{i, j+1}-2 \bar{C}_{i, j}+\bar{C}_{i, j-1}}{(\Delta Y)^{2}} . \\
& \frac{\bar{C}_{i, j}^{\prime}-\bar{C}_{i, j}}{\Delta \tau}+U_{i, j} \frac{\bar{C}_{i, j}-\bar{C}_{i-1, j}}{\Delta X}+V_{i, j} \frac{\bar{C}_{i, j+1}-\bar{C}_{i, j}}{\Delta Y}=\frac{1}{S_{C}} \frac{\bar{C}_{i, j+1}-2 \bar{C}_{i, j}+\bar{C}_{i, j-1}}{(\Delta Y)^{2}}+S_{o} \frac{\bar{T}_{i, j+1}-2 \bar{T}_{i, j}+\bar{T}_{i, j-1}}{(\Delta Y)^{2}} .
\end{aligned}
$$

And the initial and boundary conditions with finite difference scheme are;

$$
\begin{array}{llll}
U_{i, j}^{0}=0, & V_{i, j}^{0}=0, & \bar{T}_{i, j}^{0}=0, & \bar{C}_{i, j}^{0}=0, \\
U_{0, j}^{n}=0, & V_{0, j}^{n}=0, & \bar{T}_{0, j}^{n}=0, & \bar{C}_{0, j}^{n}=0, \\
U_{i, 0}^{n}=1, & V_{i, 0}^{n}=0, & \bar{T}_{i, 0}^{0}=1, & \bar{C}_{i, 0}^{n}=1, \\
U_{i, L}^{n}=0, & V_{i, L}^{n}=0, & \bar{T}_{i, L}^{n}=0, & \bar{C}_{i, L}^{n}=0,
\end{array}
$$

where, $L \rightarrow \infty$.

Here the subscripts $i$ and $j$ designate the grid points $x$ and $y$ coordinates respectively and the superscript $n$ represents a value of time, $\tau=n \Delta \tau$ where, $n=0,1,2,3, \cdots$. From the initial condition, the values of $U$ is known at $\tau=0$. Then at the end of the anytime-step $\Delta \tau$, the new temperature $\bar{T}^{\prime}$ and concentration $\bar{C}^{\prime}$, the new velocity $U^{\prime}$ at all interior nodal points may be obtained by successive application of temperature, concentration and momentum equation respectively. This process is repeated in time and provided the time-step is sufficiently small, hence $U, V, \bar{T}$ and $\bar{C}$ should eventually convergent values which approximate the steady-state 
solution of the problem.

\section{Results and Discussion}

To discuss the effects of the associated non-dimensional parameters on the flow variables, the numerical solutions of the problem are obtained by the EFDM numerical methodology with the help of a computer programming language Compaq Visual Fortran. In order to analyze the physical solution of the model, we have computed the steady numerical values of the non-dimensional Velocity $U$, Temperature $\bar{T}$, and Concentration $\bar{C}$ within the boundary layer for different values of Inclination $(\alpha)$, Grash of number $\left(G_{r}\right)$, Prandtl number $\left(P_{r}\right)$, Schmidt number $\left(S_{c}\right)$, Soret number $\left(S_{o}\right)$, and Chemical reaction parameter $\left(K_{r}\right)$. To obtain the steadystate solutions of the computation, the calculations have been carried out up to non-dimensional time $\tau=10$ to 60. Hence the velocity, temperature and concentration profile are drawn for dimensionless time $\tau=10,30$ and 60. In the Figures 2-10 $x$-axis taken as dimensionless co-ordinate variable $(Y)$ which is varies 1 to 25 and $y$-axis taken as fluid velocity, temperature and concentration respectively.

The effect of the inclination on the velocity field is presented in Figure 2. It is observed that the dimensionless velocity distribution of fluid decreases with the rise of inclination $\alpha \quad\left(=45^{\circ}, 50^{\circ}\right.$ and $\left.60^{\circ}\right)$ for different time steps. Therefore angle of inclination has a great impact on the velocity of fluids. Figure 3 represents the evolution of dimensionless velocity with $Y$ for different values of dimensionless times ( $\tau=10,30$ and 60) and Grashof number as ratio of the buoyancy to viscous force acting on a fluid was considered as $0.1,0.2$ and 0.5 . An increase in Grashof number clearly enhances profiles of velocity, therefore induces a robust speeding up in the flow. A distinct velocity shoot arises for all profiles near the boundary surface $(Y=0)$ and this is emphasized with increasing Grashof number. With increasing Grashof number the thermal buoyancy force is augmented which aids in momentum change in the boundary layer. Also the boundary layer thickness of velocity is therefore increased as well. With superior intervene of time, the velocity was found to be considerably enhanced.

The same effect on the velocity curve is found in Figure 4 that is the velocity decreases with increase of Prandtl number $P_{r} \quad(=0.71,1.0$ and 7.0). This is because Prandtl number implies the relative influence of momentum diffusion to thermal diffusion in the periphery layer regime. When $P_{r}=1$, the momentum diffusion rate surpasses thermal diffusion rate. As a result the velocity in the fluid regime will be decreased with a rise in Pr. The dimensionless velocity distribution of fluid was found to be strongly reduced for the decreasing effect of

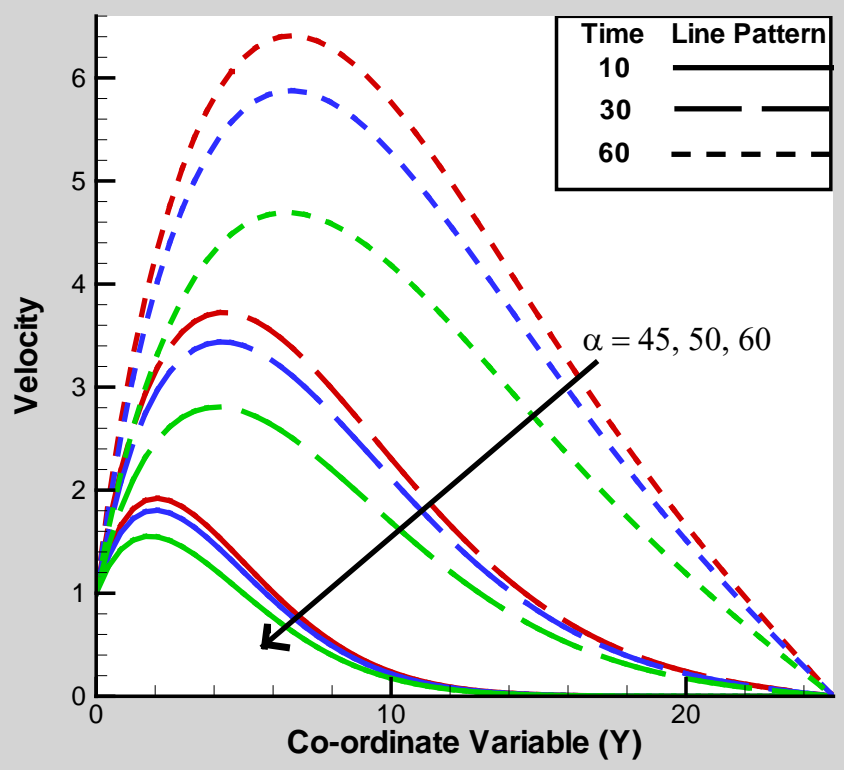

Figure 2. Velocity profiles for $G_{m}=1.0, G_{r}=0.5, S_{o}=1.0, P_{r}=0.71, S_{c}=$ $0.96, D_{f}=0.1, K_{r}=1.0$. 


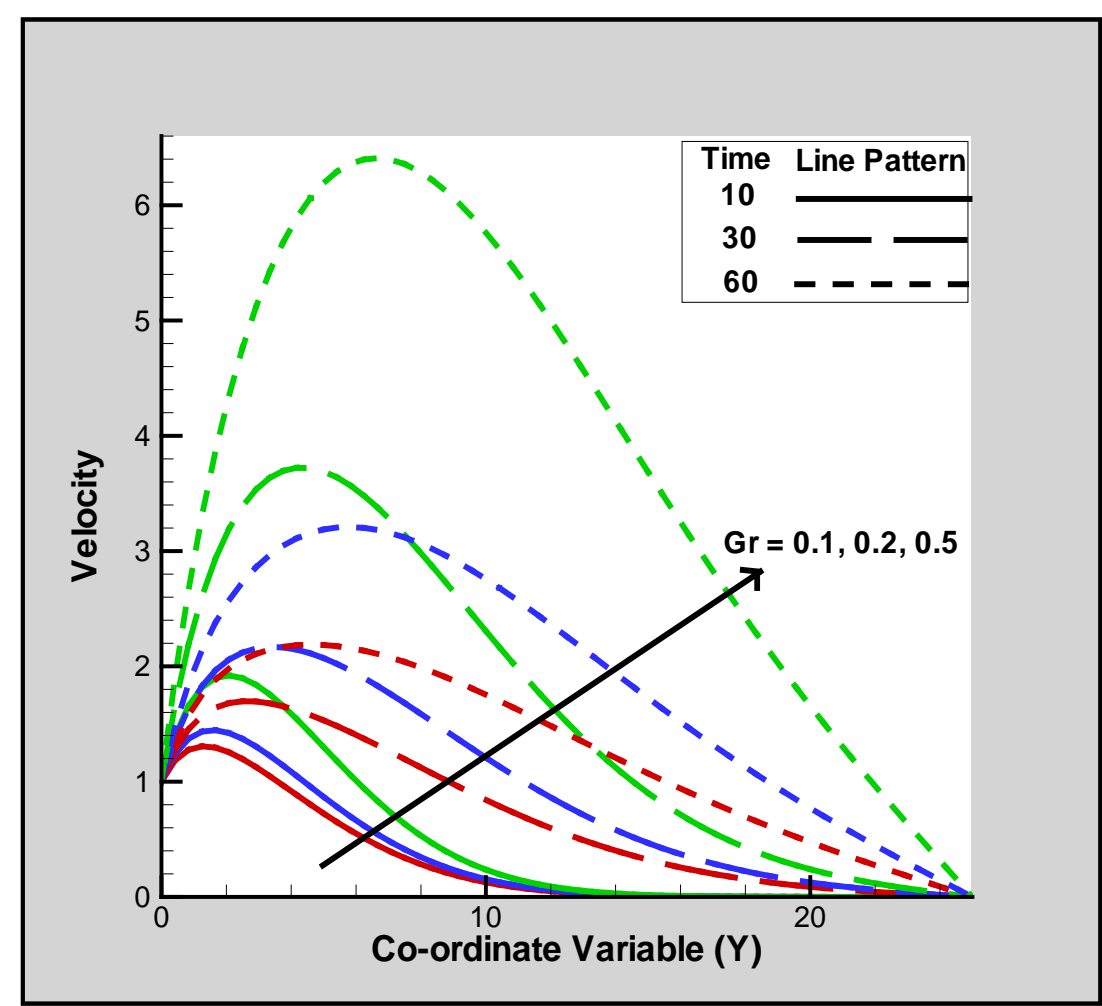

Figure 3. Velocity profiles for $G_{m}=1.0, \alpha=45^{\circ}, S_{o}=1.0, P_{r}=0.71, S_{c}=0.96, D_{f}=$ $0.1, K_{r}=1.0$.

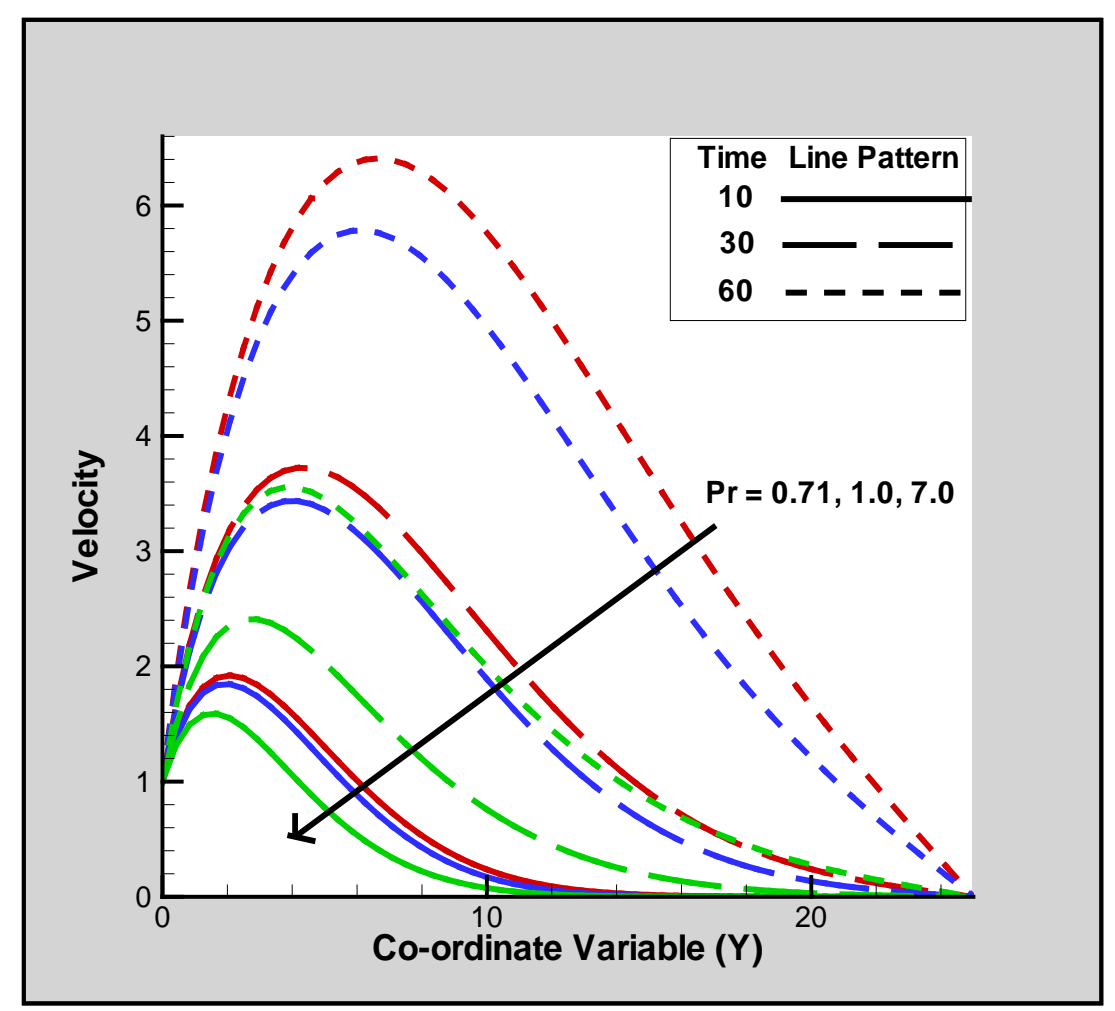

Figure 4. Velocity profiles for $G_{m}=1.0, \alpha=45^{\circ}, G_{r}=0.5, S_{o}=1.0, S_{c}=0.96, D_{f}=$ $0.1, K_{r}=1.0$. 


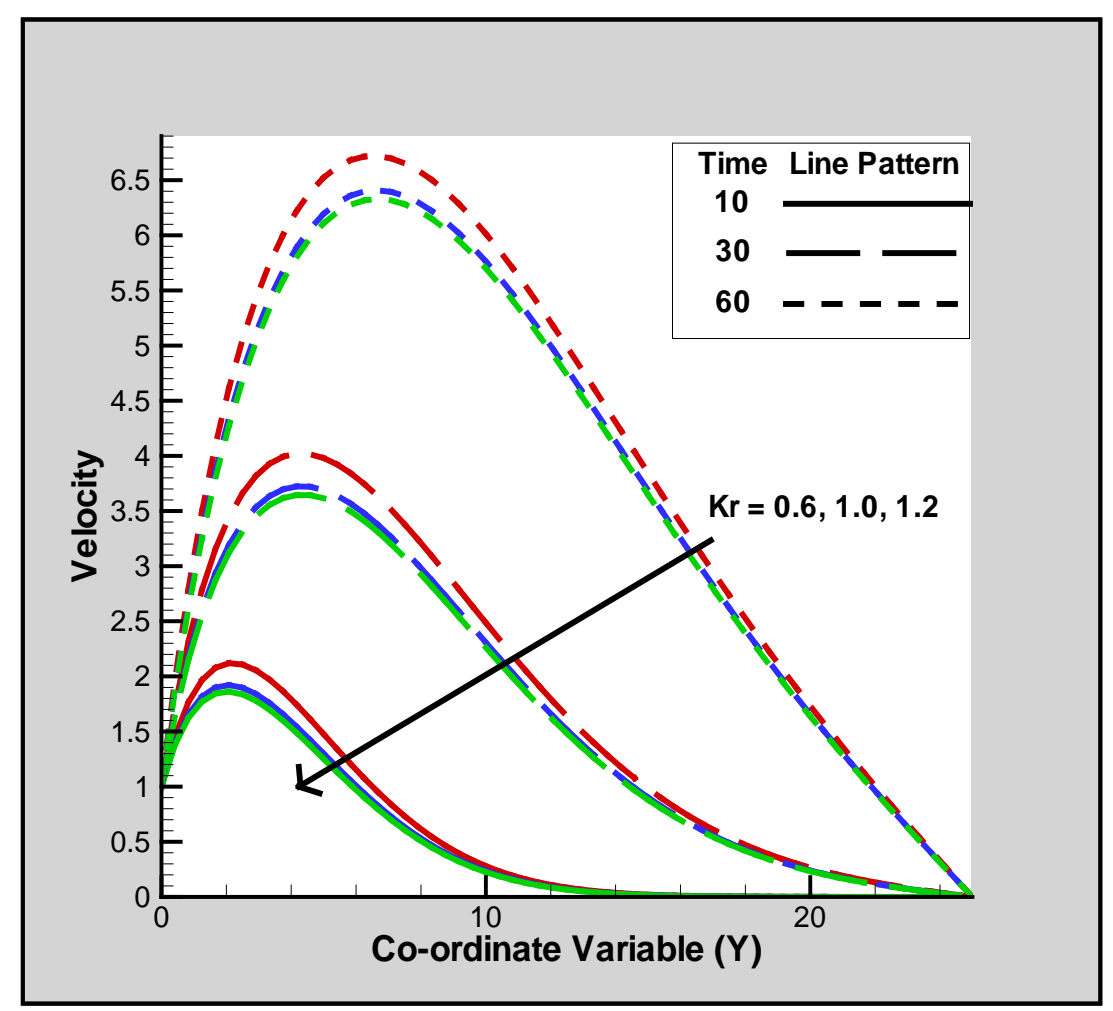

Figure 5. Velocity profiles for $G_{m}=1.0, \alpha=45^{\circ}, G_{r}=0.5, S_{o}=1.0, P_{r}=0.71, S_{c}=$ $0.96, D_{f}=0.1$.

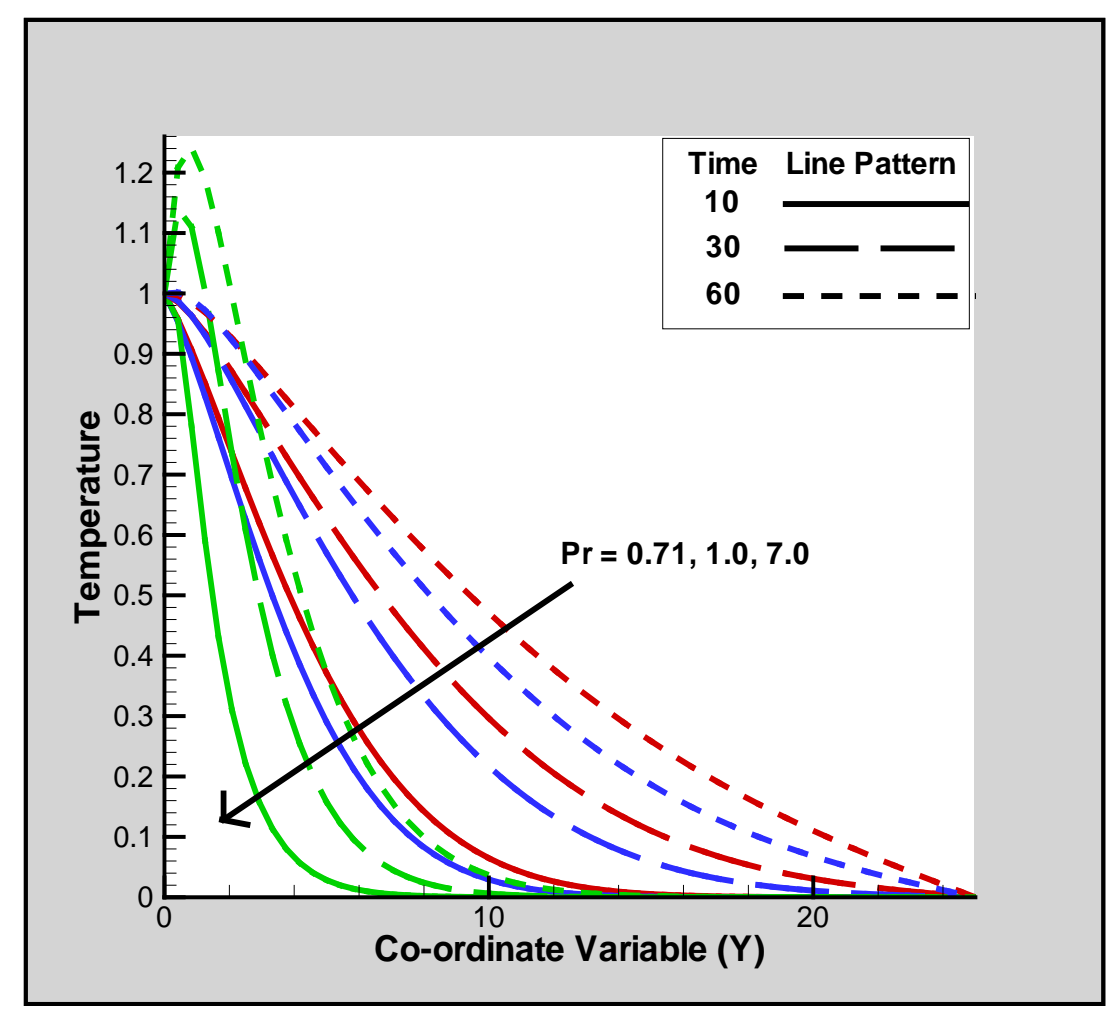

Figure 6. Temperature profiles for $G_{m}=1.0, \alpha=45^{\circ}, G_{r}=0.5, S_{o}=1.0, S_{c}=0.96, D_{f}$ $=0.1, K_{r}=1.0$. 


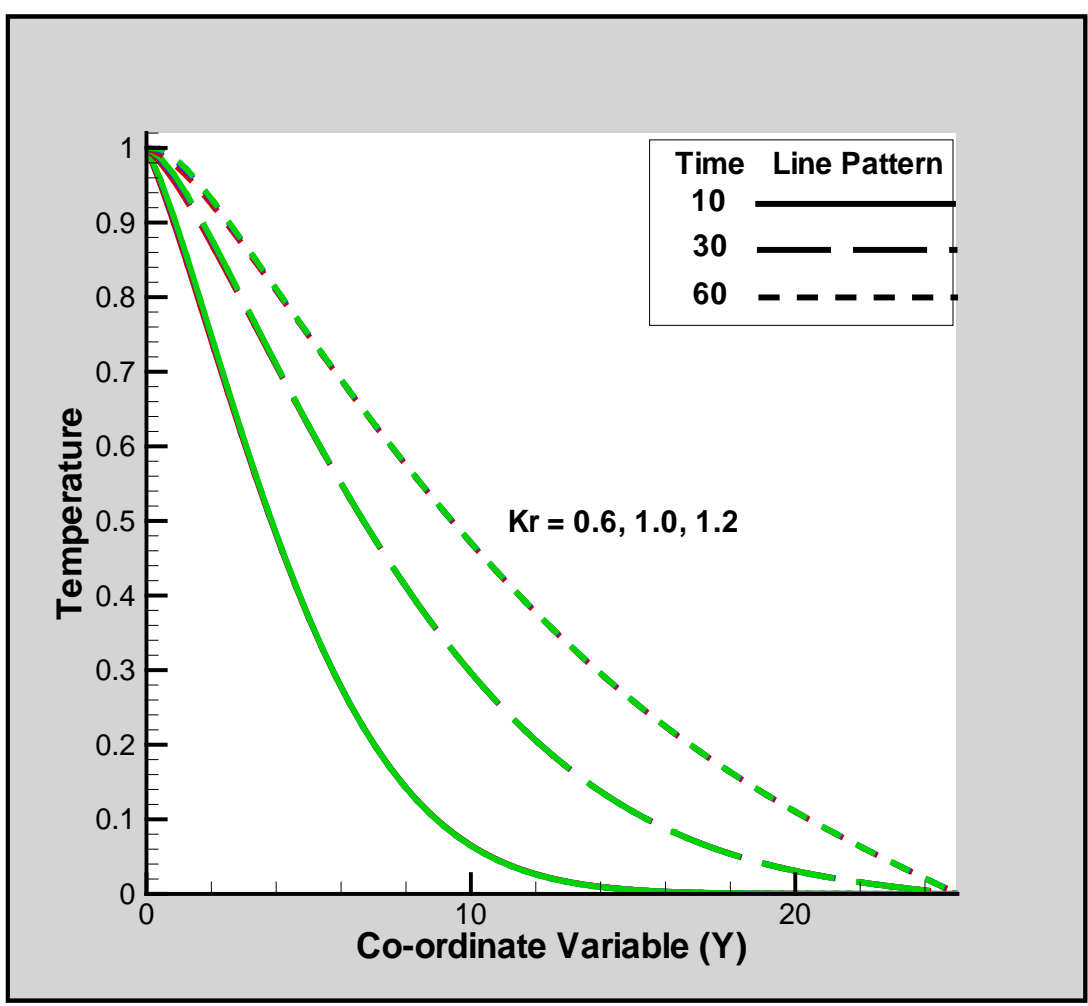

Figure 7. Temperature profiles for $G_{m}=1.0, \alpha=45^{\circ}, G_{r}=0.5, S_{o}=1.0, P_{r}=0.71, S_{c}$ $=0.96, D_{f}=0.1$.

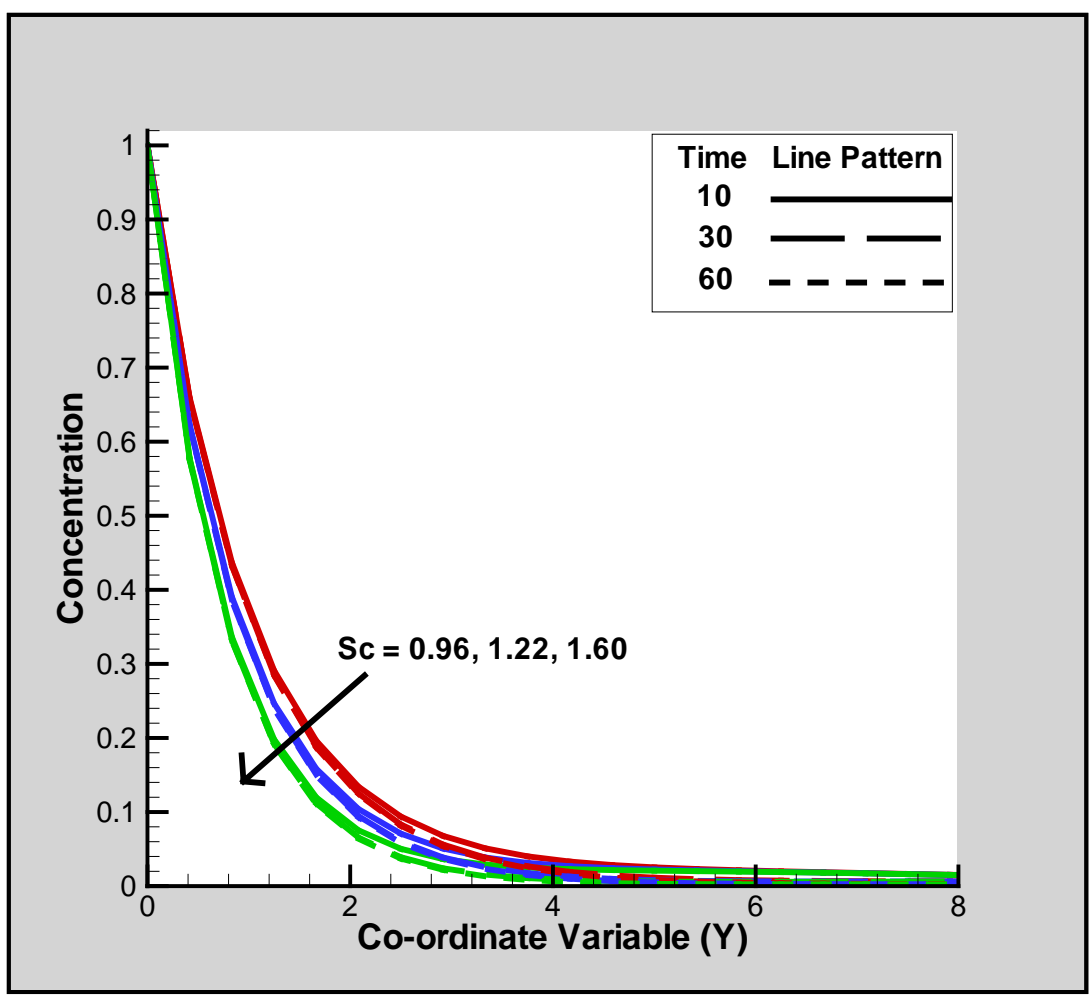

Figure 8. Concentration profiles for $\alpha=45^{\circ}, G_{r}=0.5, S_{c}=0.96, S_{o}=1.0, P_{r}=0.71$, $K_{r}=1.0, D_{f}=0.1$. 


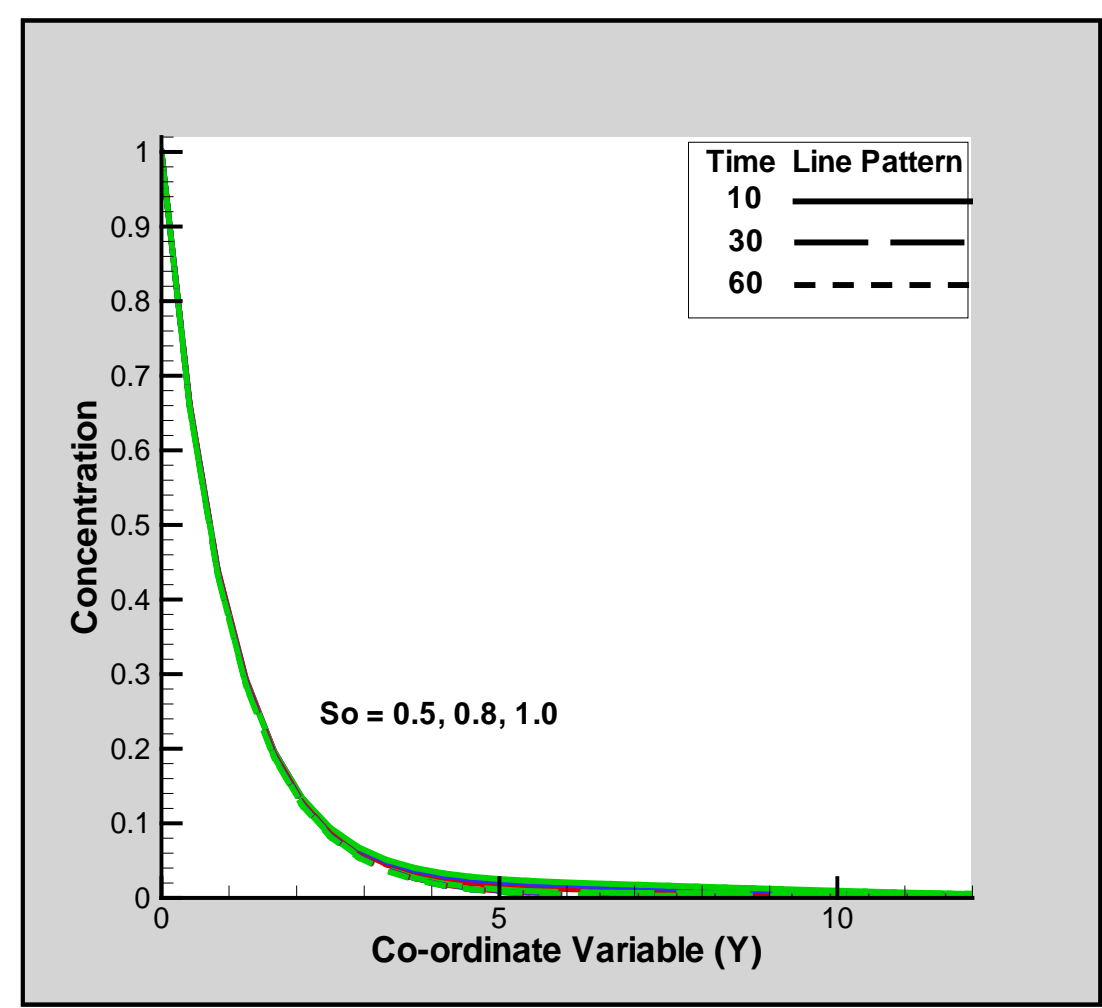

Figure 9. Concentration profiles for $G_{m}=1.0, \alpha=45^{\circ}, G_{r}=0.5, S_{c}=0.96, P_{r}=0.71$, $K_{r}=1.0, D_{f}=0.1$.

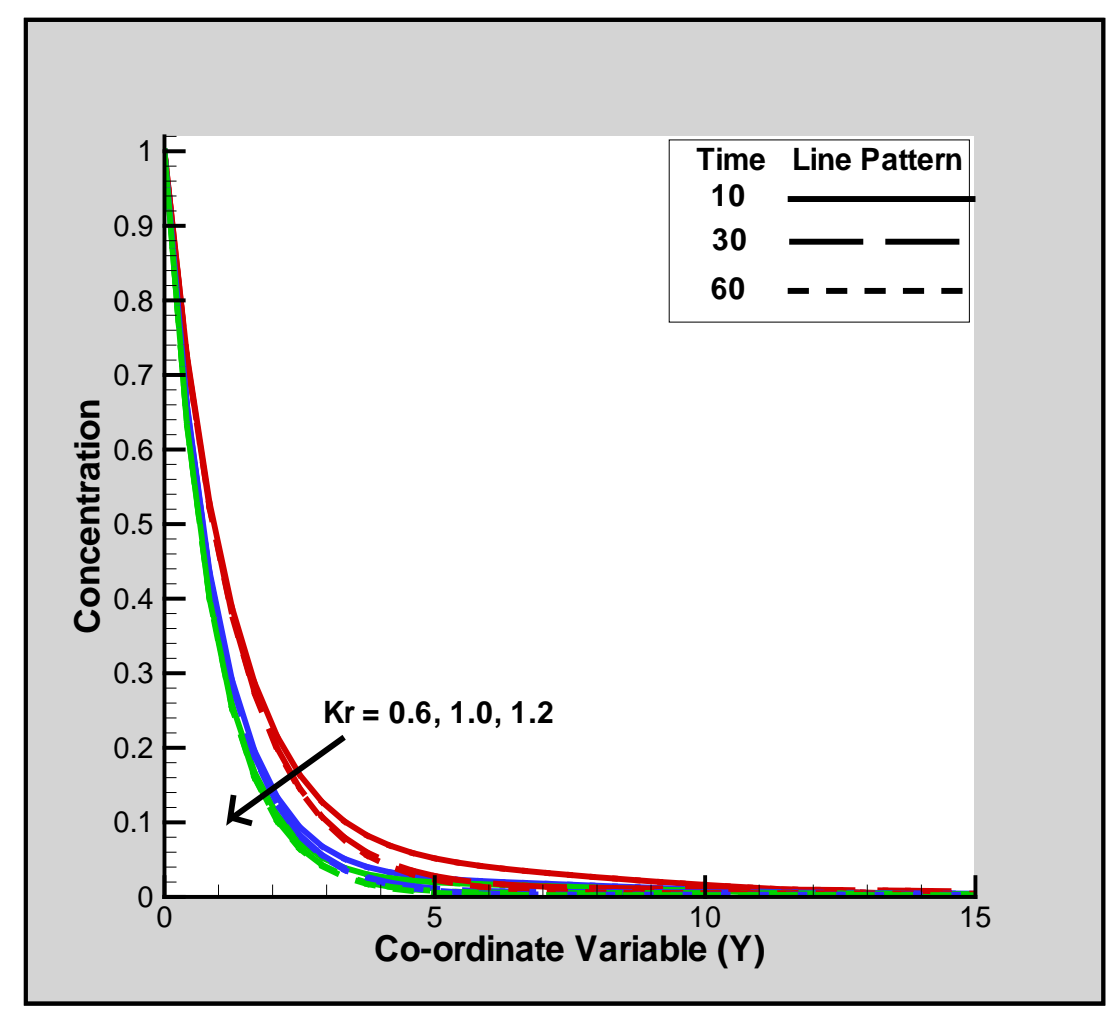

Figure 10. Concentration profiles for $G_{m}=1.0, \alpha=45^{\circ}, G_{r}=0.5, S_{o}=1.0, P_{r}=0.71$, $S_{c}=0.96, D_{f}=0.1$. 
chemical reaction parameter $K_{r}(=0.6,1.0$ and 1.2) found on Figure 5. Figure 6 shows that the steady state temperature of fluid decreases with the increase of Prandtl number $P_{r} \quad(=0.71,1.0$ and 7.0$)$. This is because the thermal boundary layer thickness decreases due to increase in this parameter. With increasing period, temperatures are yet again detected to be strongly enhanced through the boundary surface.

In Figure 7 it was observed that the fluid temperature remain unchanged with the increasing value of chemical reaction parameter $K_{r} \quad(=0.6,1.0$ and 1.2).

A decreasing effect of dimensionless mass distribution curves are observed for increasing Schmidt number $S_{C}$ in Figure 8 that is fluid concentration decreases with increase of Schmidt number $S_{C} \quad(=0.96,1.22$ and 1.60). Therefore the ratio of diffusivity of momentum i.e., viscosity and diffusivity of mass has a remarkably effects on fluid concentration. And the Schmidt number can be used to describe fluid flows in which there are instantaneous processes of momentum and mass diffusion convection. The unchanged effect of Soret number $S_{o}$ on concentration profiles are observed in Figure 9 where the mass distribution of fluid is remain same with increasing value of Soret number $S_{o}$. The ratio of thermos-diffusion and diffusion coefficient was assumed to be 0.5, 0.8 and 1.0. In Figure 10 it can be seen that the fluid concentration remains unchanged with the increasing value of chemical reaction parameter $K_{r} \quad(=0.6,1.0$ and 1.2). From the above figures we also observed that fluid behavior is varies with dimensionless time $\tau$. With the rise of dimensionless time $\tau$ the fluid velocity, temperature and concentration are gradually rises.

\section{Conclusions}

Mixed (combined) convection boundary layer flows are of great attention because of their innumerable industrial, engineering and scientific applications in heat and mass transfer. In this paper, a boundary layer analysis for mixed convection heat and mass transfer fluid flow through an inclined plate in the presence of chemical reaction and thermal diffusion is considered. The governing of boundary layer flow such as momentum, energy and mass balance is then numerically analyzed by explicit finite difference method (EFDM). The results are presented graphically with the effect of various dimensionless parameters. The significant findings obtained from the graphical observation are listed below:

- The velocity distribution decreases with the increase of inclined angle $(\alpha)$.

- The velocity distribution increases with the increase of Grashof number $\left(G_{r}\right)$.

- The velocity distribution decreases with the increase of Prandtl number $\left(P_{r}\right)$, also the temperature distribution decreases with the increase of Prandtl number $\left(P_{r}\right)$.

- The Concentration distribution decreases with the increase of Schmidt number $\left(S_{c}\right)$.

- The Concentration distributions remain unchanged with the increase of Soret number $\left(S_{o}\right)$.

- The velocity distribution and concentration distribution both are decreased with the increase of chemical reaction parameter $\left(K_{r}\right)$ while the temperature distribution remains unchanged with the variety of Chemical reaction parameter $\left(K_{r}\right)$.

\section{Acknowledgements}

Three of authors (FA, MMI and MSH) of this research paper are thankful to their M.Sc. (Applied Mathematics) thesis supervisor AI for his endless support and valuable suggestion during the preparation of the thesis as well as manuscript. All authors are also grateful to Mathematics Discipline, Khulna University, Bangladesh for outstanding facilities of Math laboratory and limitless cooperation of faculty members.

\section{References}

[1] Pera, L. and Gebhart, B. (1971) The Nature of Vertical Natural Convection Flows Resulting from the Combined Buoyancy Effects of Thermal and Mass Diffusion. International Journal of Heat and Mass Transfer, 15, 269-278. http://dx.doi.org/10.1016/0017-9310(72)90074-9

[2] Soundalgekar, V.M. and Ganesan, P. (1981) Finite-Difference Analysis of Transient Free Convection with Mass Transfer on an Isothermal Vertical Flat Plate. International Journal of Engineering Science, 19, 757-770. http://dx.doi.org/10.1016/0020-7225(81)90109-9

[3] Singh, A.K., Singh, A.K. and Singh, N.P. (2003) Heat and Mass Transfer in MHD Flow of a Viscous Fluid Past a Vertical Plate under Oscillatory Suction Velocity. Indian Journal of Pure and Applied Mathematics, 34, 429-442.

[4] Ambethkar, V. (2008) Numerical Solutions of Heat and Mass Transfer Effects of an Unsteady MHD Free Convective 
Flow Past an Infinite Vertical Plate with Constant Suction. Journal of Naval Architecture and Marine Engineering, 5, 27-36.

[5] Sivaiah, M., Nagarajan, A.S. and Reddy, P.S. (2009) Heat and Mass Transfer Effects on MHD Free Convective Flow past a Vertical Porous Plate. The ICFAI University Journal of Computational Mathematics, 2, 14-21.

[6] Umemura, A. and Law, C. K. (1990) Natural Convection Boundary Layer Flow over a Heatedplate with Arbitrary Inclination. Journal of Fluid Mechanics, 219, 571-584. http://dx.doi.org/10.1017/S0022112090003081

[7] Chamka, A. and Khaled, A.R.A. (2001) Simultaneously Heat and Mass Transfer in Free Convection. Industrial Engineering Chemical, 49, 961-968.

[8] Reddy, M.G. and Reddy, N.B. (2011) Mass Transfer and Heat Generation Effects on MHD Free Convection Flow past an Inclined Vertical Surface in a Porous Medium. Journal of Applied Fluid Mechanics, 4, 7-11.

[9] Singh, P.K. (2012) Heat and Mass Transfer in MHD Boundary Layer Flow past an Inclined Plate with Viscous Dissipation in Porous Medium. International Journal of Scientific \& Engineering Research, 3, 2229-5518.

[10] Ali, L.E., Islam, A. and Islam, N. (2015) Investigate Micropolar Fluid Behavior on MHD Free Convection and Mass Transfer with Constant Heat and Mass Fluxes by Finite Difference Method. American Journal of Applied Mathematics, 3, 157-168. http://dx.doi.org/10.11648/j.ajam.20150303.23

[11] Islam, M., Akter, F. and Islam, A. (2015) Mass Transfer Flow through an Inclined Plate with Porous Medium. American Journal of Applied Mathematics, 3, 215-220. http://dx.doi.org/10.11648/j.ajam.20150305.12

[12] Chaudhary, R.C. and Jha, A.K. (2008) Effects of Chemical Reactions on MHD Micropolar Fluid Flow past a Vertical Plate in Slip-Flow Regime. Applied Mathematics and Mechanics, 29, 1179-1194. http://dx.doi.org/10.1007/s10483-008-0907-x

[13] Muthuchumaraswamy, R. (2009) First Order Chemical Reaction on Exponentially Accelerated Isothermal Vertical Plate with Mass Diffusion. Annals Faculty of Engineering, 7, 47-50.

[14] Rajesh, V. and Varma, S.V.K. (2009) Chemical Reaction and Radiation Effects on MHD Flow Past an Infinite Vertical Plate with Variable Temperature. Far East Journal of Mathematical Sciences, 32, 87-106.

[15] Eckert, E.R.G. and Drake, R.M. (1972) Analysis of Heat and Mass Transfer. McGraw-Hill Book Company, New York.

[16] Olajuwon, B.I. (2011) Convection Heat and Mass Transfer in a Hydromagnetic Flow of a Second Grade Fluid in the Presence of Thermal Radiation and Thermal Diffusion. International Communications in Heat and Mass Transfer, 38, 377-382. http://dx.doi.org/10.1016/j.icheatmasstransfer.2010.11.006

[17] Kumar, A.G.V., Goud, Y.R. and Varma, S.V.K. (2012) Thermal Diffusion and Radiation Effects on Unsteady MHD Flow through Porous Medium with Variable Temperature and Mass Diffusion in the Presence of Heat Source/Sink. Advances in Applied Science Research, 3, 1494-1506.

[18] Ferdows, M., Khan, M.S., Bég, O.A. and Alam, M.M. (2013) Numerical Study of Transient Magneto Hydrodynamicradiative Free Convection Nanofluid Flow from a Stretching Permeable Surface. Journal of Process Mechanical Engineering, 1-16.

[19] Khan, M.S., Karim, I., Ali, L.E. and Islam, A. (2012) MHD Free Convection Boundary Layer Unsteady Flow of a Nanofluid along a Stretching Sheet with Thermal Radiation and Viscous Dissipation Effects. International Nano Letters, 2, 1-9. http://dx.doi.org/10.1186/2228-5326-2-24 


\section{Nomenclature}

$C_{p}$ : Specific heat at constant pressure

$C$ : Concentration

$D_{m}$ : Mass diffusivity

$D_{T}$ : Thermal diffusivity

$g$ : Local acceleration due to gravity

$k$ : Thermal conductivity

$T$ : Fluid temperature

$\overline{T^{\prime}}$ : Dimensionless fluid temperature

$U$ : Dimensionless primary velocity

$V$ : Dimensionless secondary velocity

$u, v$ : Velocity components along $\mathrm{x}$ and y axes respectively

$Y$ : Dimensionless coordinate variable

\section{Dimensionless parameters}

$D_{f}$ : Dufour number

$G_{r}$ : Grashof number

$G_{m}$ : Modified Grashof number

$K_{r}$ : Chemical reaction parameter

$P_{r}$ : Prandtl number

$S_{C}$ : Schmidt number

$S_{0}$ : Soret number

\section{Greek Symbols}

$\beta_{T}$ : Thermal expansion coefficient $\beta_{C}$ : Mass expansion coefficient

$\rho$ : Density of the fluid

$\mu$ : Dynamic viscosity of the fluid

$v$ : Kinematic viscosity of the fluid

$\alpha$ : Angle of inclination

$\Delta$ : Differential operator

$\delta$ : Boundary layer thickness

\section{Subscripts}

$w$ : Condition of the wall

$\infty$ : Condition of the free steam

\section{Abbreviations}

EFDM: Explicit finite difference method

MHD: Magneto hydrodynamics 